Research Article

\title{
Innovación Social en las universidades al servicio de la sostenibilidad. Circular Society Lab como estudio de caso
}

\section{Social Innovation in universities at the service of sustainability. Circular Society Lab as a case study}

\author{
Alexia Sanz-Hernández ${ }^{* *}$ y Marianna Martínez Alfaro² \\ 1,2 Departamento de Psicología y Sociología, Grupo Multidisciplinar de Socioeconomía y Sostenibilidad \\ (S33_17R), Facultad de Ciencias Sociales y Humanas, Universidad de Zaragoza \\ *Correspondencia: alexsanz@unizar.es
}

\begin{abstract}
Resumen: Muchas organizaciones públicas y privadas de todo el mundo están haciendo un gran esfuerzo para implicarse en el reto global de contribuir a las transiciones de sostenibilidad en el marco del desarrollo sostenible, colocando a la innovación en el centro de su acción. Este artículo analiza la potencialidad de los laboratorios de innovación como ecosistemas abiertos para canalizar estos esfuerzos en el contexto universitario, a partir de una revisión de la literatura y la presentación de un estudio de caso, el Circular Society Labs. El artículo contribuye aportando un marco analítico para el estudio de la relación entre innovación y sostenibilidad en las universidades, enfatizando las potencialidades de los laboratorios para integrar acciones de innovación social con educación, gestión, investigación y concienciación social para la acción e implementación de nuevos modelos socioeconómicos basados en la sostenibilidad y la circularidad.
\end{abstract}

Palabras clave: innovación social, universidades, transiciones de sostenibilidad, circularidad, estudio de caso.

\begin{abstract}
Digitalization of public life has a serious impact on the development of social and labor relations. In spite of the fact that digitalization has many positive effects, we can point out that the issue of employment precarization is coming to the fore and it is reflected in the growth of vulnerability and social insecurity among workers. This trend is manifested to the fullest extent in the Russian society. In this regard our paper considers theoretical and practical issues of employment precarization in Russia in the context of its rapid digitalization. In the course of our analysis we clarify the concept of precarious employment, identify digitalization trends and the extent and causes of instability in the social and labor sphere. In particular, we have found out that more and more people are involved in unstable labor relations against the background of the rapidly forming digital environment in the country.
\end{abstract}

Keywords: social innovation, universities, sustainability transitions, circularity, case study.

\section{Introducción}

El marco de desarrollo sostenible se construye a partir de la multidimensional noción de sostenibilidad, caracterizada por su complejidad y polisemia desde sus orígenes. El llamado Informe Brundtland (WCED, 1987) recoge la conceptualización posiblemente más consensuada 
y aceptada en las diferentes esferas, incluida las Naciones Unidas. Ésta llama a una movilización social y global hacia un desarrollo que atienda a las necesidades del presente sin comprometer la capacidad de las generaciones futuras para cubrir sus propias necesidades. A partir de entonces, la noción abierta de 'necesidades' ha avivado un rico debate sin precedentes que expresa diferentes posiciones ideológicas sobre la sostenibilidad planetaria (Lamberton, 2005; Lafferty \& Langhelle, 1999) y sobre la satisfacción de las necesidades de las generaciones futuras condicionada al consumo de fuentes agotables y recursos limitados en el presente.

El establecimiento en 2015 por parte de la ONU de los 17 Objetivos de Desarrollo Sostenible (ODS), en la llamada Agenda 2030, es otro hito relevante que marca las pautas para avanzar en las dimensiones económicas, sociales y medioambientales de un mundo sostenible, en un intento por superar una de las principales críticas y desavenencias en el marco del desarrollo sostenible (DS), a saber, el desacuerdo sobre lo que debe ser sostenido (sostenibilidad planetaria, sostenibilidad de las personas, sostenibilidad de las economías...) y la propia operatividad de la sostenibilidad (su medición y su traducción en objetivos y fases concretas para su consecución) (Sachs, 1999).

Para avanzar en los ODS se requieren dos dinámicas fundamentalmente, el compromiso de todos los actores y el pleno desarrollo de la innovación en todos los campos. Los laboratorios de innovación social son posiblemente el marco que mejor combina ambas dinámicas.

Los laboratorios son lugares donde nacen las ideas, por lo que cada vez más los contextos académicos albergan estas figuras para contribuir a generar conocimiento de una forma innovadora. Por su parte, los laboratorios sociales son plataformas ideadas para abordar retos que presentan tres rasgos, su carácter social, su naturaleza experimental y su enfoque sistémico. Estos entornos se han multiplicado en las últimas décadas (Hassan, 2014), aunque el enfoque de experimentación social y de participación ciudadana no es nuevo.

Así pues, sostenibilidad e innovación son dos importantes desafíos que también las universidades han asumido, pero en los que se debe profundizar más. Por ello contextualizamos este artículo en los esfuerzos de las universidades desde un enfoque de campus multi-rol (Arroyo, 2015), es decir, considerando los diferentes campos o dominios que le son inherentes: el organizativo y estratégico (Lozano et al. 2015; Ramos et al. 2015), el educativo (Educación para un Desarrollo Sostenible, EDS) (UNESCO, 2012; Ávila et al., 2017), el investigador (van den Heiligenberg, 2017) y, finalmente, el de transferencia, también llamado 'tercera misión' (Purcell, 2019).

Este artículo contribuye de varias maneras. En primer lugar, presenta el estado del arte actualizado en relación con los laboratorios de innovación social centrados en la sostenibilidad y el papel que juegan las universidades en ellos, para destacar los aspectos relevantes que los caracterizan y proponer un marco analítico para el estudio de la innovación para la sostenibilidad en las universidades.

Segundo, muestra un estudio de caso, el Circular Society Labs de la Universidad de Zaragoza (España) (CSLUZ), como ejemplo de los esfuerzos para lograr un ecosistema sostenible basado en la innovación. Este caso contribuye en primer lugar a resaltar las potencialidades de los labs como marco para el desarrollo de experiencias de aprendizaje desde un enfoque amplio e interdisciplinar de sostenibilidad en un momento en el que todavía el DS es percibido como un complemento, más que como un aspecto inherente de la Educación Superior (Ávila et al., 2017). Segundo, aporta un nuevo enfoque para superar algunas de las barreras que se han identificado en la contribución de las universidades en el logro de los ODS, como la confusión acerca del concepto de DS y sus prácticas, la escasez de amplitud en la noción de DS y la escasa centralidad de la sostenibilidad en las disciplinas universitarias, el currículo académico y la investigación (Ávila et al., 2017; Leal et al., 2017). Y tercero, analiza los diferentes roles que está jugando la universidad focalizándose en una de las más importantes brechas observadas en la gestión de las prácticas de sostenibilidad en las universidades para el logro de un SD (desarrollo sostenible): la inclusión activa de los estudiantes. 
El artículo se estructura de la siguiente manera. En la sección dos se presenta el estado del arte y el marco analítico propuesto para el análisis de la innovación social en las universidades; en la tercera se describe el estudio de caso, destacando los objetivos, ámbitos, estructura y metodología del laboratorio de innovación presentado. En la sección cuarta se presentan los resultados poniendo de relieve el potencial que los laboratorios de innovación pueden tener en el contexto universitario. El quinto punto de discusión aporta algunas recomendaciones en forma de decálogo y limitaciones y, finalmente en la sección sexta se concluye.

\section{Estado del arte y marco analítico}

\subsection{El debate en torno a las transiciones de sostenibilidad y el desarrollo sostenible}

Las iniciativas de sostenibilidad han venido cuestionando el modo en que se hacían las cosas (Thompson \& Green, 2005). La propia noción de sostenibilidad y especialmente la de DS, tampoco han estado exentas de crítica. Esta se ha centrado en las contradicciones internas del término (Dresner, 2002; Jabareen, 2008), en el cuestionamiento de su intención 'real' y las incompatibilidades en su propuesta central, es decir, en la interacción entre desarrollo económico, los estilos de vida que sustentan las sociedades contemporáneas y la protección medioambiental (López-Pardo, 2015).

Para justificar y avanzar en el DS se han planteado posicionamientos diversos que reflejan diferente grado de optimismo y confianza en la capacidad e ingenio humano para innovar y satisfacer las necesidades. Por ejemplo, Cox y Ziv (2005) contraponen cierto maltusianismo frente a enfoques antropocéntricos optimistas como el denominado "entrepreneurialism", que busca compatibilizar e integrar los objetivos sociales y los ecológicos combinando economicismo con optimismo tecnológico, además de la propia sostenibilidad ambiental. Incluso algunos autores plantean un modelo de sostenibilidad normativo y abierto, cuyo contenido se determine por deliberación pública (Arias Maldonado, 2004).

En este debate complejo también surgen concepciones competidoras acerca del tipo de transformación hacia la que encaminar la acción y los aspectos que deben enfatizarse en ellas: los cambios estructurales de la producción y consumo (D'Alisa et al., 2015), las innovaciones tecnológicas y la política 'progresiva' (O’Brien, 2011), o bien, las redes de movimientos cívicos o iniciativas de acción social para construir y abarcar cambios más amplios (Stirling, 2015).

Pese a todo este debate acerca de qué clase de transformaciones plantear y cómo avanzar en ellas, los autores coinciden en la capacidad del concepto para animar las transiciones (Axon, 2020), y en la conveniencia de entender el concepto de sostenibilidad como un proceso de reflexión y acción hacia objetivos que no pueden alcanzarse, pero que guían a las sociedades hacia el cambio y la mejora (Williams et al., 2017).

En esta corriente, compatibilizando y resolviendo operativamente las contradicciones que el propio concepto plantea, las universidades están participando de manera desigual con propuestas de sostenibilidad (Leal et al., 2019). Estas se plasman en dos niveles de acción, el organizacional avanzando hacia los campus sostenibles, y el global con el que se han relacionado las aportaciones para la implementación de nuevos modelos socioeconómicos circulares (economía verde, economía circular, bioeconomía), de consumo y de producción (por ejemplo, Verhoef, 2018; Verhoef et al., 2020).

\subsection{La institucionalización de la innovación para la sostenibilidad en las universidades}

Las universidades han sido consideradas un espacio bien posicionado tanto para educar a los futuros líderes bajo la lente de la sostenibilidad (Elliott \& Wright, 2013), como para co-crear soluciones que den respuesta a las necesidades sociales en el marco del DS.

Los primeros espacios universitarios de experimentación y co-creación bajo la denominación de laboratorios (labs) surgieron desde la innovación tecnológica, a menudo bajo 
la denominación de living labs, y con el propósito de incorporar a los usuarios finales en el proceso de innovación, diseño y co-producción desde las primeras etapas (Baelden, 2015; Evans et al., 2015).

Con el tiempo los labs se han desarrollado en ámbitos enormemente diversos, siendo uno de ellos la educación superior (Booponoyeng Bayuo et al., 2020). Sus aproximaciones participativas han tenido diferentes niveles de éxito (Disterheft et al., 2014) y de impacto transformador, y en la mayoría de ocasiones las experiencias han perseguido una innovación incremental (creación de valor sobre un producto o servicio ya existente gracias a la incorporación de mejoras), más que a una innovación radical (creación de nuevos productos o servicios) (Leminen, 2015).

El origen de los labs vinculado a la innovación tecnológica y el mayor número de experiencias en ese ámbito discurren paralelos a la apertura en su conceptualización, conforme el enfoque sociotécnico ha ido poniendo de relieve cómo los factores sociales influyen en la configuración de las innovaciones tecnológicas, y a la inversa. Así, los labs han avanzado incrementándose el peso de lo social, redefiniendo sus objetivos y estructuras, equiparando el protagonismo de los usuarios de la innovación al del resto de participantes en las experiencias de innovación (investigadores, diseñadores, decisores, etc.), y consecuentemente, involucrando a estos desde las primeras etapas del proceso co-creador (Almirall et al., 2012). Se ha pasado de atender a la innovación para el desarrollo de productos, testeo de tecnologías y servicios o mejora de servicios digitales (Coetzee et al., 2012) a innovaciones en numerosos ámbitos para desarrollar capacidades y resiliencia comunitaria, mejorar la vida cotidiana de las personas, desarrollar bienestar y calidad de vida de los ciudadanos, testear servicios a los ciudadanos y mejorar los entornos de vida. El usuario pasa de ser objeto (procesos de innovación centrados en el usuario), a asumir su responsabilidad y agencia en la co-creación (Liedtke et al., 2012).

La aproximación desde Innovación Social ha ido ganando terreno con celeridad, resultando difícil para la literatura académica recoger y cuantificar las muchas experiencias que surgen en todos los niveles y entornos. En concreto, en el ámbito universitario español estas iniciativas se están multiplicando en los últimos años (algunos liderados por universidades con actividad, que se recogen en la Figura 1.), con intentos de articulación de redes, como la denominada Red de Universidades por la Innovación Pública impulsada por el Medialab de la Universidad de Granada, que trabaja en este momento por la innovación pública desde la esfera académica (Romero-Frías \& Robinson-García, 2017).

Figura 1. Institucionalización de los laboratorios de innovación social en las universidades españolas

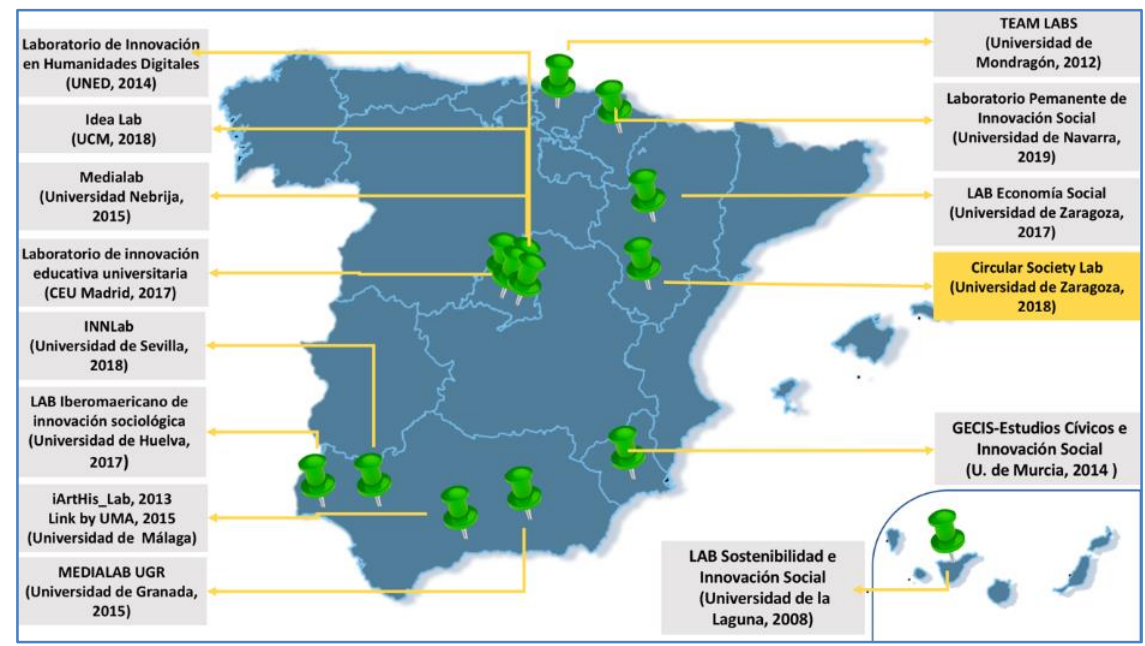

Fuente: elaboración propia a partir de las webs institucionales, 2020. 


\subsection{Marco analítico para el estudio de la innovación en sostenibilidad en universidades}

La conceptualización de los laboratorios de innovación en el tiempo refleja la comentada evolución desde lo tecnológico a lo social. En este proceso, la literatura ha caracterizado los laboratorios de innovación (labs, hub, living labs, fab, labs, etc...) como un método de innovación (Dell'Era \& Landoni, 2014; Dutilleul et al., 2010), un set de herramientas de innovación abierta (Kviselius et al., 2009), como plataformas (presenciales o virtuales) de experimentación (Schuurman et al., 2011), o como un tipo de redes de innovación abierta (Romero \& Molina, 2012).

A efectos de esta investigación, entendemos los laboratorios de innovación como ecosistemas abiertos (por el flujo de ideas que provienen de fuentes tanto internas como externas a la organización), con continuidad o no en el tiempo (iniciativas aisladas vs. estructuras institucionalizadas), que tienen como objetivo resolver problemas de diferente grado de complejidad (atendiendo a los niveles, ámbitos y campos que contemplan), y que implican a diferentes actores afectados (stakeholders) asumiendo diversos roles y responsabilidades en la interacción que se establece entre ellos (Figura 2.).

Figura 2. Marco analítico para el estudio de la innovación en sostenibilidad en las universidades.

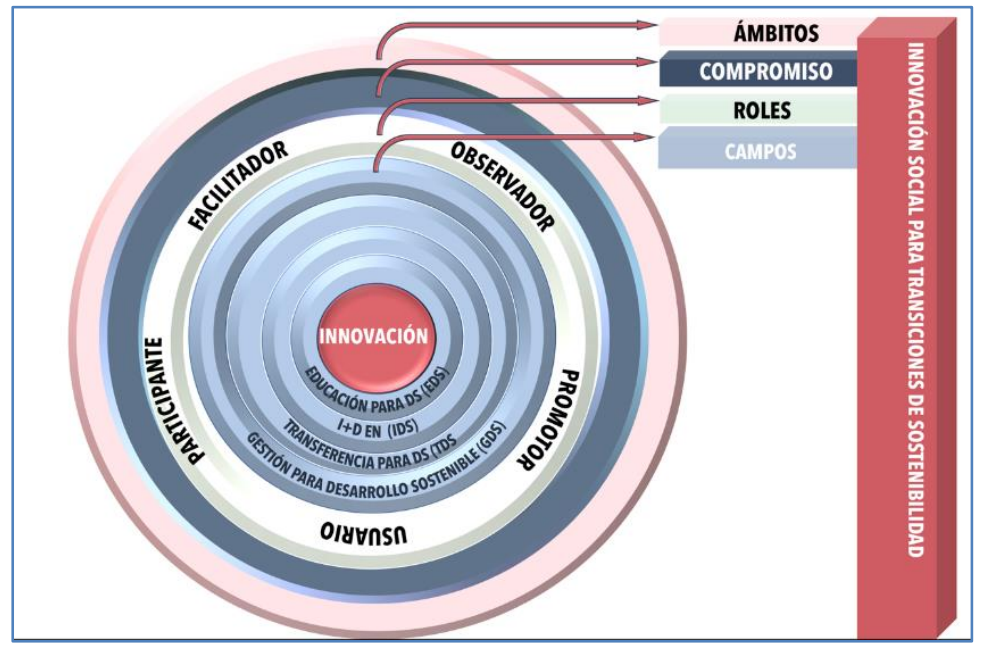

Fuente: elaboración propia, 2020.

El marco analítico propuesto permite analizar si, como cabría esperar, se ha producido una mayor amplitud en los ámbitos de acción atendidos, un incremento del compromiso con la innovación, la asunción de roles proactivos y de mayores responsabilidades individuales, colectivas y organizacionales (niveles de rol), y finalmente, una integración de los dominios o campos que, por su naturaleza, atienden las universidades: educación, investigación, transferencia (a veces denominada tercera misión), y gestión organizacional.

De todas estas cuestiones, únicamente ampliamos aquí una breve descripción de la tipología de roles de las universidades que hemos distinguido en el análisis: 1) Observador (mero espectador o investigador). 2) Facilitador, contribuye generando un marco y creando las condiciones favorables para el desarrollo de experiencias de innovación social en proyectos impulsados tanto interna como externamente. 3) Participante, colabora en los procesos de innovación social como un stakeholder más aportando información y sumando ideas. 4) Promotor, asume la iniciativa, incorpora el enfoque en su estrategia y lidera los procesos y experiencias. A nivel institucional, esto se ha plasmado por ejemplo en la red de oficinas verdes que la mayoría de las universidades han incorporado para trabajar en el ámbito de la sostenibilidad como parte de su estrategia organizacional. Y, 5) Usuario activo, toma parte y forma parte del grupo co-creador de las soluciones a los problemas y retos de innovación. En 
este caso, las universidades afrontan problemas y necesidades internas/externas que son puestas de manifiesto y resueltas por la propia comunidad universitaria.

Cada uno de ellos puede referirse a tres niveles: individual (investigadores, estudiantes, profesores a título personal), grupal (según el grupo formal al que pertenece, grupos de investigación, equipos docentes, grupos de estudiantes, de PAS, etc.) e institucional/organizacional (relativo a estructuras universitarias formales, campus, centros o unidades organizativas).

\section{Estudio de caso: Circular Society Labs de la Universidad de Zaragoza (CSLUZ)}

El CSLUZ se diseñó en 2018 como un ecosistema de innovación abierto para coordinar diferentes experiencias y proyectos en el marco universitario usando novedosas metodologías participativas y teniendo como objetivo principal el de involucrar a la comunidad universitaria y a la sociedad en general en retos de sostenibilidad, desarrollo sostenible e implementación de modelos de producción y consumo circulares y sostenibles.

CSLUZ no es una iniciativa aislada y se asienta en la estructura formal de una cátedra institucional. Nace en el marco de la colaboración público-privada entre la universidad y el ámbito empresarial (Grupo Térvalis). Esta ubicación permite plantear el lab con un horizonte temporal de continuidad y conformarse como la suma de proyectos que van surgiendo a lo largo del tiempo. En el lab se sigue una metodología de desarrollo de proyectos ('proyecto a proyecto') en un ciclo iterativo e incremental en el que cada proyecto atraviesa diferentes fases (Figura 3.).

Figura 3. Metodología de desarrollo de proyectos

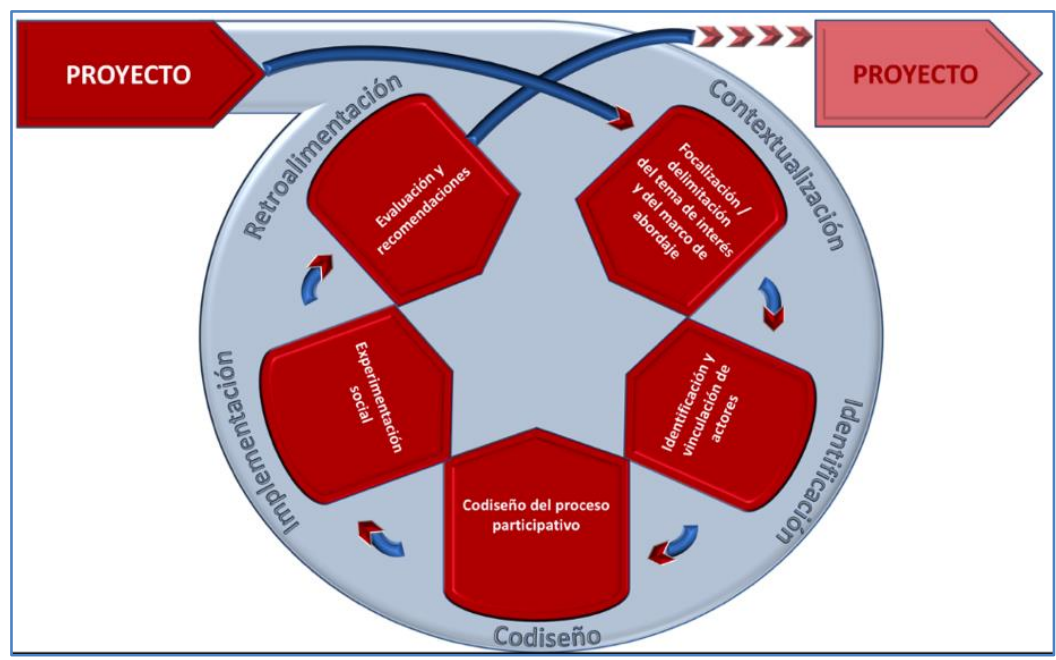

Fuente: elaboración propia, 2020.

El hecho de que el lab esté enraizado en el ámbito académico como factor de innovación pública, ha permitido establecer alianzas estables con diferentes redes (universitarias o no), y experimentar de manera transversal, siempre siguiendo cinco principios potenciadores de la participación de la ciudadanía: innovación cívica, diseño abierto, colaboración, multidisciplinariedad y justicia global.

Los proyectos planteados hasta el momento se enfocan a innovación incremental en diferentes ámbitos de interés que en parte moldean la estructura organizativa del lab. CSLUZ coloca la sostenibilidad en el corazón de su actividad e incorpora elementos relacionados con la bioeconomía circular. El modelo circular aboga por un mayor y mejor uso de materias primas renovables no fósiles, el uso eficiente de los recursos y por nuevos estilos de producir y 
consumir (también prosumo). Se han diseñado siete áreas que responden a los ámbitos de la bioeconomía circular (Sanz-Hernández et al., 2019), cuatro de ellos son de carácter temático y tres son transversales (Figura 4.).

Figura 4. Ámbitos de interés y estructura de CSLUZ

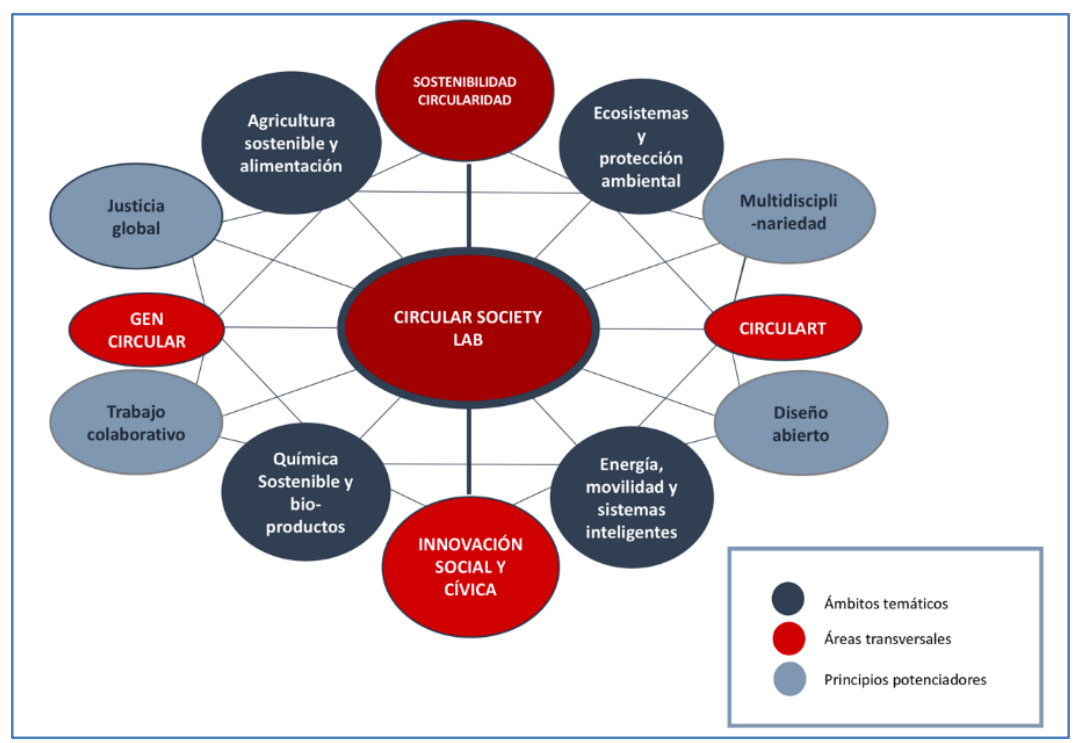

Fuente: elaboración propia, 2020.

Las áreas transversales de género y creatividad se concretan en los programas GenCircular (incorporando explícitamente una línea de trabajo de género como marco interpretativo y perspectiva de análisis de los cambios) y CirculArt (de marcado perfil socioecológico y centrado en profundizar en las estrechas conexiones entre la creatividad y la sostenibilidad a partir de experiencias artísticas (Covaleda \& Sanz-Hernández, 2020).

La tercera área transversal es la de innovación social que actúa de aglutinadora de la iniciativa. La innovación reside en las personas (innovación cívica), va de la mano del aprendizaje y la creatividad y debe estar al servicio de las necesidades sociales. Los productos de la innovación tienen un impacto social que debe medirse, precisamente para dar valor social a lo que creamos y conocer en qué medida contribuimos a hacer una sociedad mejor y un planeta sostenible.

El Circular Society Labs es un 'lugar' para la experimentación, la creatividad y la exploración de nuevas formas de conocimiento y de experiencia. Del mismo modo facilita la implementación de diversidad de metodologías que potencian la generación de inteligencia colectiva y la toma de decisiones. En la sociedad analógica, los labs representan formas empáticas de construir conocimiento aplicado siguiendo hojas de ruta presencial. A lo largo de su año de andadura, CSLUZ ha realizado acciones vinculadas a procesos participativos, publicaciones y alianzas con entidades vinculadas a la Bioeconomía y Economía Circular, Medio Ambiente o Agenda 2030, también a iniciativas que han surgido a propósito del COVID19 (para ampliar información ver https://circularsocietylabs.unizar.es/).

Precisamente ante el escenario del COVID-19, han proliferado los mecanismos de participación que catapultan a las TIC como factores a tomar en cuenta para los tiempos venideros. En la sociedad digital, los labs de "talento distribuido" representan nuevas formas de participación ciudadana en la creación de comunidad en línea. En el Circular Society Labs se entienden ambas modalidades como espacios abiertos. Una como 'hub' físico y otra en redes (distribuido) para conectar y recombinar el conocimiento generado en la cultura digital, incorporando la tecnología a los procesos de generación del conocimiento, de interacción social, y de participación pública. Por tanto, el diseño se ha convertido en un espacio "híbrido" donde 
se entremezclan las formas de generar conocimiento, partiendo del aprendizaje para desaprender. La transformación digital invita a desprogramar y repensar la necesidad de utilizar metodologías novedosas que ayudan a fomentar la innovación social.

\section{Resultados}

La corta experiencia en el CSLUZ nos permite aportar los siguientes resultados relevantes para destacar la adecuación de los laboratorios de innovación social en el marco universitario y hacer propuestas para un diseño que permita compatibilizar en los marcos institucionales la enseñanza, investigación y transferencia, en consonancia con las experiencias que otros autores están poniendo de relieve. Se describen a continuación las principales potencialidades.

\subsection{Capacidad de adaptación a contextos educativos de diferente nivel de formalidad: Curriculum formal/informal/oculto}

Los proyectos de innovación docente en el CSLUZ rompen con los límites de la formalidad y diluyen las fronteras entre los diferentes tipos de currículo. Tanto los planes de estudio formales, como el currículum informal (e incluso el oculto), pueden brindar muchas oportunidades para involucrar a los estudiantes con ESD (Hopkinson et al., 2008; Lozano et al., 2013) en experiencias de gestión universitaria en los campus, programas de investigación o proyectos de innovación docente, por ejemplo. Además, su rol puede llegar a ser relevante y central.

La presencia y relevancia de la educación para el DS en el plan de estudios formal suele depender tanto de los intereses del profesorado como del grado de compromiso de la institución universitaria con la sostenibilidad. Durante décadas la noción de sostenibilidad en educación superior se ha vinculado a ámbitos científicos y tecnológicos y ha estado ausente en las disciplinas sociales y humanísticas (Pierce, 2014). Los labs permiten explorar nuevas propuestas de curriculum en cualquier disciplina, demostrando las potencialidades de proyectos en marcos de informalidad e incluso de currículum oculto.

\subsection{Desarrollo de experiencias integrales}

Todos los proyectos desarrollados catalizan experiencias de aprendizaje y oportunidades de trabajo inter y transdisciplinario, e interorganizacional, con igual o mayor potencial de impacto interno y externo que las experiencias académicas curriculares formales, al basarse en un aprendizaje activo (Bonwell \& Eison, 1991). De ahí que sea importante implicar a los estudiantes en los diferentes proyectos integrados, uniendo educación, investigación, gestión y transferencia (Okayama, 2019).

\subsection{Capacidad para dar forma a la interdisciplinaridad/transdisciplinaridad}

A pesar del reconocimiento global de la necesidad de una EDS desde enfoques interdisciplinares (Wals, 2014; UNESCO, 2016), del papel y responsabilidad de las instituciones de educación superior a través de la investigación y la enseñanza interdisciplinaria y de la alta eficacia de las experiencias transdisciplinares para desarrollar competencias imprescindibles para el DS (Rieckmann, 2012), no es fácil incorporar la aproximación holística, la interdisciplinariedad, la transversalidad y la coordinación. Hay consenso acerca de que el trabajo interdisciplinario es vital para ofrecer soluciones a los problemas del desarrollo insostenible (Shiel et al., 2016), pero su materialización es sin duda uno de los puntos más limitantes dentro de las organizaciones para el desarrollo de proyectos. 


\subsection{Co-creación de experiencias de aprendizaje}

Uno de los principios clave de la sostenibilidad subraya la necesidad de que las personas a través de su participación, estén en el corazón de las transformaciones sostenibles. Sin embargo, colocar la innovación social en el centro de la labor universitaria puede resultar tarea complicada por las numerosas barreras en ámbitos institucionalizados (como el universitario). Por ejemplo, en el dominio docente los métodos tradicionales de enseñanza suelen estar enfocados para tareas rutinarias (Ávila et al., 2017). Ante esto, los labs suponen terrenos de experimentación que van cuestionando y erosionando lentamente la manera tradicional en que se vienen haciendo las cosas y abriendo camino para la apertura institucional a nuevos procesos y metodologías.

\subsection{Contribuir a forjar agentes de cambio}

Desde 2018 se ha visto a nivel mundial un incremento exponencial del compromiso social, público y privado con la sostenibilidad (Axon, 2020), pero se sigue observando una gran brecha entre el nivel discursivo y el práxico. Los labs son espacios facilitadores de prácticas creativas y participativas que estimulan la reflexión, la creación, la comunicación y también la acción. Son un puente para que las instituciones educativas incorporen un enfoque educativo transformador y un cambio en la perspectiva en relación con los actores.

Las prácticas de sostenibilidad en las universidades suelen diseñarse e implementarse siguiendo la lógica dominante "top-down", escaseando las iniciativas centradas en los estudiantes en particular, como agentes de cambio (Elliott \& Wright, 2013). CSLUZ facilita experiencias que desvelan nuevos caminos incluyendo prácticas sociales y modos diferentes de ver el mundo, con los que todos los actores pueden comprometerse para conseguir impactos cada vez mayores y sostenidos en el tiempo.

CSLUZ nos está permitiendo conocer más acerca de cómo los jóvenes se comprometen con estilos de vida sostenibles y cómo se conectan con los problemas de sostenibilidad (Wolf \& Moser, 2011). La cuestión de responsabilidad, asunción de la agencia y compromiso social, tanto en el plano individual como en el colectivo $u$ organizacional, pueden además actuar como motivadores para los otros. Es lo que Axon (2020) denomina el principio del "othering" o el fenómeno "I will if you will".

\subsection{Potencia para crear mensajes de sostenibilidad de mayor impacto social}

El trabajo colectivo del lab y las formas comunicativas adoptadas (Djordjevic \& Cotton, 2011), pueden incrementar el impacto social de las acciones del lab. Por ejemplo, la experiencia Mail me Art (Covaleda \& Sanz-Hernández, 2020) ha permitido demostrar la potencialidad del arte para comunicar mensajes de sostenibilidad (Gombrich, 2003). Esta aproximación innovadora aglutinó a 30 estudiantes de arte universitarios y jóvenes artistas, en un espacio expresivo para la reflexión, la concienciación y la crítica/protesta en torno a la sostenibilidad. La experiencia estimuló la deliberación individual y colectiva, facilitó el aprendizaje y activó el deseo de los participantes por implicarse en la transformación societal.

\section{Discusión}

CSLUZ es un ejemplo multidisciplinar de integración de iniciativas de DS en diferentes dominios con acciones que involucran planes de estudio, investigación y proyectos con las partes interesadas y la sociedad.

Los laboratorios de innovación en el marco universitario (es el caso de CSLUZ) están permitiendo la adquisición de competencias clave en la EDS como la capacidad para reflexionar sobre modelos individuales y culturales, para pensar críticamente descubriendo las 
contradicciones inherentes a los modelos de vida sostenible (producción y consumo), la asunción de la responsabilidad individual y el propio papel en la comunidad global, la posesión de creatividad para idear soluciones y eventos futuros y su capacidad para comunicar los mensajes de sostenibilidad.

En el marco del esfuerzo en innovación que se está realizando desde las universidades para adaptarse a los cambios sociales, la experiencia CSLUZ deja un decálogo de recomendaciones para atender a las demandas sociales.

1. Atención al cambio continuo y cultura de innovación-reflejo de lo social.

2. Acciones híbridas que integren procesos en diferentes niveles (individual, comunitario y organizacional).

3. Cadenas de alianzas para generar valor en la esfera pública.

4. Espacios de experimentación físicos o virtuales facilitadores de la participación de la ciudadanía.

5. Acciones efervescentes y capaces de dar respuesta también a demandas específicas o procesos puntuales.

6. Capilaridad para un flujo de la información a todos los niveles y en todas las direcciones.

7. Catalización para el fomento de sinergias.

8. Aceptación de los desaciertos, como paso inherente al éxito futuro de una innovación.

9. Orientación de la acción hacia escenarios de futuro (Thinking Futures).

10. Orientación a la mejora de la gestión pública con la ineludible colaboración de todos los actores (y niveles de roles).

En general, se ve necesario un cambio de paradigma hacia una universidad en la que se integre en armonía la formación, la investigación, la transferencia, y su propia gestión organizacional; no obstante, existen numerosas barreras internas y externas, algunas de las cuales ya se han puesto de manifiesto. Evidenciamos aquí solo dos de ellas. La primera es la complejidad en la búsqueda de la interdisciplinariedad, que requiere aunar intereses, capacidades y motivaciones individuales muy dispares y de numerosos actores en proyectos compartidos, cuya co-creación y co-diseño además es complicado de gestionar.

La segunda es la falta de asunción de las universidades de su responsabilidad y la conciencia de su axial papel en las transiciones de sostenibilidad. Prestar atención a las demandas de los actualmente más desfavorecidos, mientras se anticipan las necesidades venideras (Anand \& Sen, 1994), requiere que la universidad asuma con responsabilidad un rol más activo que vaya más allá del de observador o facilitador, como paso previo para el abordaje de las grandes cuestiones del DS, como la equidad en sus diversas dimensiones relacionadas con el DS (intra o intergeneracional, e interterritorial) y escasamente atendida, según la experiencia en CSLUZ.

No obstante, estas y otras muchas de las barreras existentes no deben condicionar la experimentación con esas nuevas formas colaborativas de afrontar los retos institucionales y globales a partir de la innovación.

\section{Conclusiones}

El artículo aborda áreas de investigación poco estudiadas aportando un marco analítico que puede facilitar una mejor comprensión de las formas en que se están desarrollando los laboratorios de innovación social centrados en la sostenibilidad, los aspectos relevantes que los caracterizan y el papel que pueden jugar las universidades. Además, presenta un estudio de caso (CSLUZ) que ejemplifica la potencialidad de los labs para promover las transiciones de sostenibilidad, en concreto desde el marco de la economía circular.

CSLUZ es un ecosistema de innovación abierto, que ha incorporado elementos de innovación social poniendo a las personas en el centro de los procesos; alineando las acciones con la Agenda 2030, estableciendo alianzas con actores sociales vinculados a la economía 
circular y generando conocimiento a través de los marcos de la gobernanza global capaz de articular trabajo colaborativo y cooperativo con agentes del territorio.

La sostenibilidad todavía no se ha incorporado plenamente en el marco estratégico institucional ni en los planes de estudio de la educación superior. CSLUZ da relevancia a la noción de sostenibilidad, trabaja a partir de las visiones que sobre la sostenibilidad tiene la comunidad universitaria, especialmente los estudiantes, y da a la noción de DS una visión amplia y posibilitista, enlazando reflexión con acción para el cambio.

El estudio ha detectado la potencialidad de las experiencias desde laboratorios de innovación en la ESD, lo que nos permite aportar claves teóricas y metodológicas para ver cómo se pueden focalizar experiencias integradas de enseñanza, investigación, transferencia o gestión basadas en la innovación social en diferentes límites y planos de estudio, para seguir trabajando competencias como sistemas de pensamiento, pensamiento futuro, compromiso personal, motivación para la acción, sostenibilidad, comunicación y acción.

Los proyectos en CSLUZ se ubican en la intersección de la educación para la sostenibilidad, la innovación social y la investigación para la implementación de modelos socioeconómicos circulares. El estudio de caso presentado se distingue de otros similares no solo por este objetivo sino también por el enfoque integrador, posibilista, transdisciplinar e inclusivo tanto de actores como de metodologías. La experiencia asume el desafío de diseñar nuevas experiencias de aprendizaje, de investigación y comunicativas contextualizando el momento de la crisis sanitaria global en el que nos encontramos, que ha puesto a prueba igualmente las metodologías de enseñanza y co-creación, y explicitado las necesidades de nuevos canales para la innovación social, educativa y organizacional.

Esta experiencia puede alentar a los académicos y profesores investigadores de sostenibilidad o de las organizaciones a expandir su horizonte metodológico para capturar los procesos que estudian o enseñan. Infundir experiencias creativas y participativas de aprendizaje en el plan de estudios de educación superior puede ayudar a los estudiantes y a la sociedad a reflexionar y a reconocer el papel de los humanos en el planeta y a trabajar la innovación (creatividad) para abordar los grandes desafíos asociados con la sostenibilidad. La incorporación de nuevos métodos de aprendizaje y nuevos enfoques para estimular la creatividad son relevantes en respuesta a las crecientes demandas de innovación en las sociedades presentes y futuras en el marco de DS.

Una línea de continuidad de este estudio sería profundizar en el poder de los labs para comunicar e impactar en la sociedad dado que esta parte no se ha podido trabajar todavía en profundidad. Así, el trabajo en CSLUZ y los resultados presentados en este artículo se complementarían con el análisis de los efectos de la comunicación de los mensajes de sostenibilidad que contienen las acciones realizadas desde el lab y su impacto en el ámbito empresarial y social.

\section{Referencias}

Almirall, E., Lee, M. \& Wareham, J. (2012). Mapping living labs in the landscape of innovation methodologies. Technology innovation management review, 2(9), 12-18. DOI: https://doi.org/10.22215/timreview/603

Anand, S. \& Sen, A. (1994). Sustainable Human Development: Concepts and Priorities. UNDP Human Development Report Office. Disponible en: https://ssrn.com/abstract=2294664

Arias, M. (2004). Sustentabilidad y democracia. Hacia una articulación democrática del principio de sustentabilidad. Revista Española de Ciencia Política, 11, 121-148. 
Arroyo, P. (2015). A new taxonomy for examining the multi-role of campus sustainability assessments in organizational change. Journal of Cleaner Production, 140, 1763-1774. DOI: https://doi.org/10.1016/j.jclepro.2015.08.100

Ávila, L.V., Leal, W., Brandli, L.L., Macgregor, C., Molthan-Hill, P., Ozuyar, P.G. \& Moreira, R.M. (2017). Barriers to innovation and sustainability at universities around the world. Journal of Cleaner Production, 164, 1268-1278. DOI: https://doi.org/10.1016/j.jclepro.2017.07.025

Axon, S. (2020). The socio-cultural dimensions of community-based sustainability: Implications for transformational change. Journal of Cleaner Production, 266, $121933 . \quad$ DOI: https://doi.org/10.1016/j.jclepro.2020.121933

Bonwell, C.C. \& Eison, J.A. (1991). Active Learning: Creating Excitement in the Classroom. Barcelona: ERIC Publications.

Booponoyeng Bayuo, B., Chaminade, C. \& Göransson, B. (2020). Unpacking the role of universities in the emergence, development and impact of social innovations - A systematic review of the literature. Technological Forecasting E Social Change, 155, 120030. DOI: https://doi.org/10.1016/j.techfore.2020.120030

Coetzee, H., du Toit, I.M. \& Herselman, M. (2012). Living labs in South Africa: an analysis based on five case studies. The Electronic Journal for Virtual Organizations and Networks, 14, 1-29.

Covaleda I. \& Sanz-Hernández. (2020). Mail me Art. Catálogo sobre Arte y sostenibilidad. Zaragoza: Universidad de Zaragoza.

Cox, W. \& Ziv, J.C. (2005). Dimensions of sustainability. Conservatoire National des Arts et Metiers, Paris 3rd International SIIV Conference Bari, Italy September 22-24, $2005 . \quad$ En https://www.researchgate.net/publication/242301087 Dimensions of sustainability [consulta el 12 de Mayo, 2020].

D'Alisa, G., Demaria, F. \& Kallis, G. (2015). Degrowth: A Vocabulary for a New Era. London: Routledge.

Dell'Era, C. \& Landoni, P. (2014). Living lab: a methodology between user-centred design and participatory design. Creativity and Innovation Management, 23(2), 137-154. DOI: https://doi.org/10.1111/caim.12061

Disterheft A, Caeiro S, Azeiteiro UM. \& Filho, WL. (2014). Sustainable universities - a study of critical success factors for participatory approaches. Journal of Cleaner Production, 106, 11-21. DOI: https://doi.org/10.1016/j.jclepro.2014.01.030

Djordjevic, A. \& Cotton, DRE. (2011). Communicating the sustainability message in higher education institutions. International Journal of Sustainability in Higher Education, 12(4), 381-394. DOI https://doi.org/10.1108/14676371111168296

Dresner, S. (2002). The Principles of Sustainable Development. London: Earthscan Publications.

Dutilleul, B., Birrer, F.A.J. \& Mensink, W. (2010). Unpacking European living labs: analyzing innovation's social dimensions. Central European journal of public policy, 4(1), 60-85.

Elliott, H. \& Wright, T. (2013). Barriers to sustainable universities and ways forward: a Canadian students' perspective. The $3 \mathrm{rd}$ World Sustainability Forum, Sciforum, Suića. DOI: https://doi.org/10.3390/wsf3-f006

Evans J, Jones R, Karvonen A, Millard L. \& Wendler J. (2015). Living labs and co-production: university campuses as platforms for sustainability science. Current Opinion in Environmental Sustainability, 16, 1-6. DOI: https://doi.org/10.1016/j.cosust.2015.06.005 
García, N. (2009). El arte como laboratorio de la sociología (y a la inversa). Exit Book, Revista semestral de libros de arte y cultura visual, 10, 1-7.

Gombrich, E. (2003). Los usos de las imágenes. Estudio sobre la función del arte y la comunicación visual. Barcelona: Debate.

Hassan, Z. (2014). The Social Labs Revolution: A New Approach to Solving Our Most Complex Challenges. San Francisco: Berrett-Koehler Publishers.

Hopkinson, P., Hughes, P. \& Layer, G. (2008). Sustainable graduates: linking formal, informal and campus curricula to embed education for sustainable development in the student learning experience, Environmental Education Research, 14(4), 435-454. DOI: https://doi.org/10.1080/13504620802283100

Jabareen, Y. (2008). A new conceptual framework for sustainable development. Environmental Development and Sustainability, 10(2), 179-192. DOI: https://doi.org/10.1007/s10668-006-9058-z

Kviselius, NZ., Andersson, P., Ozan, H. \& Edenius, M. (2009). Living labs as tools for open innovation. Communications \& Strategies, 74, 490-504.

Lafferty, WM. \& Langhelle, O. (1999). Towards sustainable development: on the goals of development - and the conditions of sustainability. Houndmills: MacMillan Press Ltd. DOI: https://doi.org/10.1057/9780230378797

Lamberton, G. (2005). Sustainable sufficiency-an internally consistent version of sustainability. Sustainable development, 13(1), 53-78. DOI: https://doi.org/10.1002/sd.245

Lambrechts, W., Mula, I., Ceulemans, K. \& Molderez. I. (2013). The integration of competences for sustainable development in higher education: an analysis of bachelor programs in management. Journal of Cleaner Production, 48, 65-73. DOI: https://doi.org/10.1016/j.jclepro.2011.12.034

Leal, W. (ed.) (2015). Transformative approaches to sustainable development at universities: working across disciplines. Berlin: Springer. DOI: https://doi.org/10.1007/978-3-319-08837-2

Leal, W., Jim Wu, YC., Brandli, LL., Avila, LV., Azeiteiro, MA., Caeiro, S. \& Madruga, LRRG. (2017). Identifying and overcoming obstacles to the implementation of sustainable development at universities. Journal of Integrative Environmental Sciences, 14, 93-108. DOI: https://doi.org/10.1080/1943815X.2017.1362007

Leal, W., Skouloudis, A., Londero Brandli, L., Lange Salvia, A. \& Rayman-Bacchus, L. (2019). Sustainability and procurement practices in higher education institutions: Barriers and drivers. Journal of Cleaner Production, 231, 1267-1280. DOI: https://doi.org/10.1016/j.jclepro.2019.05.202

Leminen, S., Nyström, A.G. \& Westerlund, M. (2015). A typology of creative consumers in living labs. Journal of Engineering and Technology Management, 37, 6-20. DOI: http://dx.doi.org/10.1016/j.jengtecman.2015.08.008

Liedtke C, Welfens MJ, Rohn H. \& Nordmann J. (2012). Living Lab: user-driven innovation for sustainability. International Journal of Sustainability in Higher Education, 13(2), 106118. DOI: $\underline{\text { https://doi.org/10.1108/14676371211211809CrossRefGoogle Scholar }}$

López-Pardo, I. (2015). On sustainable development and sustainability: conceptualization and review. Barataria. Revista Castellano-Manchega de Ciencias Sociales, 20, 111-128. DOI: http://dx.doi.org/10.20932/barataria.v0i20.16

Lozano, R., Lozano, F.J., Mulder, K., Huisingh, D. \& Waas, T. (2013), Advancing higher education for sustainable development: international insights and critical reflections. Journal of Cleaner Production, 48, 3-9. DOI: https://doi.org/10.1016/j.jclepro.2013.03.034 
Lozano, R., Ceulemans, K., Alonso-Almeida, M., Huisingh, D., Lozano, F., Waas, T., Lambrechts, W., Lukman, R. \& Huge, J. (2015). A review of commitment and implementation of sustainable development in higher education: results from a worldwide survey. Journal of Cleaner Production, 108, 1-18. https://doi.org/10.1016/j.jclepro.2014.09.048

Massek, T. (2017). Living labs in architecture as innovation arenas within higher education institutions. Energy Procedia, 115, 383-389. DOI: https://doi.org/10.1016/j.egypro.2017.05.035

Pierce, A. (2014). Aesthetic mediation and the politics of technology: (re)New(ed) strategies for a critical theory. Critical Horizons, 15(1), 69-81. DOI: https://doi.org/10.1179/1440991713Z.00000000021

Purcell, WM. (2019). Universities as the engine of transformational sustainability toward delivering the sustainable development goals "Living labs" for sustainability. International Journal of Sustainability in Higher Education, 20, 1343-1357. DOI: https://doi.org/10.1108/IJSHE-02-2019-0103

Ramos, T.B., Caeiro, S., Van Hoof, B., Lozano, R., Huisingh, D. \& Ceulemans, K. (2015). Experiences from the implementation of sustainable development in higher education institutions: environmental management for sustainable universities. Journal of Cleaner Production, 106, 3-10. DOI: https://doi.org/10.1016/j.jclepro.2015.05.110

Rieckmann, M. (2012). Future-oriented higher education: which key competencies should be fostered through university teaching and learning? Futures, 44, 127-135. DOI: https://doi.org/10.1016/j.futures.2011.09.005

Romero, D. \& Molina, A. (2012). Collaborative networked organizations and customer communities: value co-creation and co-innovation in the networking era. Production Planning \& Control, 22(5-6), 447-472. DOI: https://doi.org/10.1080/09537287.2010.536619

Romero-Frías, E. \& Robinson-García, N. (2017). Laboratorios sociales en Universidades: Innovación e impacto en Medialab UGR. Comunicar, 51, 29-38. DOI: https://doi.org/10.3916/C51-2017-03

Sachs, W. (1999). Sustainable development and the crisis of nature: on the political anatomy of an oxymoron. En: F. Fischer \& M. Hajer (eds.), Living with Nature: Environmental Politics as Cultural Discourse (pp. 23-42). Oxford: Oxford University Press.

Sanz-Hernández, A., Esteban, E. \& Garrido, P. (2019). Transition to a bioeconomy: Perspectives from Social Sciences. Journal of Cleaner Production, 224, 107-119. DOI: https://doi.org/10.1016/j.jclepro.2019.03.168

Shiel, C., Leal Filho, W., Do Paço, A. \& Brandli, L. (2016). Evaluating the engagement of universities in capacity building for sustainable development in local communities. Evaluation and Program Planning, 54, 123-134. DOI: $\underline{\text { https://doi.org/10.1016/j.evalprogplan.2015.07.006 }}$

Schuurman, D., De Moor, K., De Marez, L. \& Evens, T. (2011). Living lab research approach for mobile TV. Telematics and Informatics, 28, 271-282. DOI: https://doi.org/10.1016/j.tele.2010.11.004

Stake, R.E. (1995). The Art of Case Study Research. London: Sage.

Stirling, A. (2015). Emancipation transformations: from controlling 'the transition' to culturing plural radical progress. En: I. Scoones, M. Leach \& P. Newell (eds.), The Politics of Green Transformations (pp. 5467) London: Routledge.

Thompson, R. \& Green, W. (2005). When sustainability is not a priority: an analysis of trends and strategies. International Journal of Sustainability in Higher Education, 6, 7-17. DOI: https://doi.org/10.1108/14676370510573104

UNESCO. (2012). Shaping the education of tomorrow report on the UN decade of education for sustainable development, abridged, DESD Monitoring and Evaluation. Paris: UNESCO. 
- (2016). Education for Sustainable Development. Paris: UNESCO. Disponible en: http://www.unesco.org/new/en/education/themes/leading-the-international-agenda/educationfor-sustainable-development/education-for-sustainable-development

Van den Heiligenberg, H. (2017). A habitat for sustainability experiments: Success factors for innovations in their local and regional contexts. Journal of Cleaner Production, 169, 204-215. DOI: https://doi.org/10.1016/j.jclepro.2017.06.177

Vare, P. \& Scott, W. (2007). Learning for a change: exploring the relationship between education and sustainable development. Journal of Education for Sustainable Development, 1(2), 191-198. DOI: https://doi.org/10.1177/097340820700100209

Verhoef, L.A. (2018). De Campus als Living Lab voor de Circulaire Economie. Stuttgart: Delft University of Technology, Hochschule für Technik Stuttgart.

Verhoef, L.A., Bossert, M., Newman, J., Ferraz, F., Robinson, ZP., Agarwala, Y., Wol, PIII, Jiranek, P. \& Hellinga, C. (2020). Towards a learning system for University Campuses as Living Labs for sustainability. En: W. Leal Filho, A. Lange Salvia, R. W. Pretorius, L. Londero Brandli, E. Manolas, F. Alves, U. Azeiteiro, J. Rogers, C. Shiel \& A. Do Paco (eds.), Universities as Living Labs for Sustainable Development: Supporting the Implementation of the Sustainable Development Goals (pp. 135-149). Cham, Switzerland: Springer.

Wals, A. E. J. (2014). Sustainability in higher education in the context of the UN DESD: a review of learning and institutionalizing processes. Journal of Cleaner Production, 62, 8-15. DOI: https://doi.org/10.1016/j.jclepro.2013.06.007

Williams, A., Kennedy, S., Phillipp, F. \& Whiteman, G. (2017). Systems thinking: a review of sustainability management research. Journal of Cleaner Production, 14, 866-881. DOI: https://doi.org/10.1016/j.jclepro.2017.02.002

Winter, J., Cotton, D., Grant, V. \& Hopkinson, P. (2015). The university as a site for transformation around sustainability. International Journal of Innovation and Sustainable Development, 9(3/4), 303-320.

Wolf, J. \& Moser, S.C. (2011). Individual understandings, perceptions, and engagement with climate change: insight from in-depth studies across the world. WIREs Climate Change, 2, 547-569. DOI: https://doi.org/10.1002/wcc.120

World Commission on Environment and Development (WCED). (1987). Our common future. The Brundtland Report. Oxford: Oxford University Press.

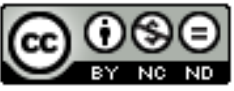

(C) Attribution-NonCommercial-NoDerivatives 4.0 International (CC BY-NC-ND 4.0)

https://creativecommons.org/licenses/by-nc-nd/4.0/ 